\title{
Manifestaciones abdominales de las anomalías del Situs Ambiguous en el adulto. A propósito de cuatro casos
}

\author{
Dres. Gonzalo Corral $G^{(1)}$, Andrés Labra $W^{(2)}$, Giancarlo Schiappacasse $F^{(3)}$.
}

1. Becado de Radiología, Universidad Mayor. Chile.

2. Radiólogo, Hospital Barros Luco Trudeau, Clínica Alemana de Santiago. Chile

3. Radiólogo, Facultad de Medicina. Clínica Alemana de Santiago - Universidad del Desarrollo. Chile

\section{Situs Ambiguous abnormalities in adults. A study of four cases}

\begin{abstract}
Anomalies Situs (ASIT) has been described mainly in the pediatric population and there is little information on this in adults.

These are very rare abnormalities; in the case of Situs Ambiguous (SAMB) in adults, there have only been a few published case series. SAMB, also called heterotaxy or heterotaxy Syndrome, is defined as the abnormal or ambiguous positioning of organs and vessels, that could also be associated with morphological alterations characteristic of each organ. There are two main groups within SAMB: polysplenia and asplenia. This publication present a specific literature review, with emphasis on the classification of adult ASIT and abdominal findings in cases of SAMB. It will be complemented clinically and radiologically with four cases of abdominal tomographic imaging studies.
\end{abstract}

Keywords: Heterotaxy, Polysplenia, Situs ambiguus.

Resumen: Las Anomalías del Situs (ASIT) han sido descritas principalmente en la población pediátrica y existe escasa información sobre éstas en el adulto.

Son anomalías muy raras; en el caso del Situs Ambiguous (SAMB) en el adulto sólo se han publicado pocas series de casos. SAMB, también llamado heterotaxia o Síndrome de heterotaxia, es definido como la posición anormal o ambigua de los órganos y vasos, que además pueden asociarse a alteraciones morfológicas características de cada órgano. Existen dos grandes grupos dentro de los SAMB: Poliesplenia y Asplenia. En la presente publicación se expone una revisión bibliográfica específica, la cual tiene énfasis en clasificación de las ASIT del adulto y los hallazgos abdominales en los casos de SAMB. Será complementada clínica e imaginológicamente con cuatro casos de estudios tomográficos abdominales.

Palabras clave: Heterotaxia, Poliesplenia, Situs ambiguous.

Corral G, et al. Manifestaciones abdominales de las anomalías del Situs Ambiguous en el adulto. A propósito de cuatro casos. Rev Chil Radiol 2013; 19(1): 38-43.

Correspondencia: Dr. Andrés LabraW. / labraw@yahoo.com

Trabajo recibido el 23 de diciembre de 2012, aceptado para publicación el 19 de febrero de 2013.

\section{Introducción}

Las Anomalías del Situs (ASIT) han sido descritas principalmente en la población pediátrica. En contraste, existe escasa información en la literatura sobre estas entidades en adultos. Esto puede ser explicado, al menos en parte, a que existen patologías fuertemente asociadas a estas anomalías las cuales impiden a muchos pacientes llegar a la edad adulta, tales como cardiopatías congénitas, inmunodeficiencias y obstrucción intestinal. Por otro lado, cuando los pacientes adultos presentan una ASIT, lo hacen con sintomatología de carácter leve y/o oligoasintomáticas, razón por la cual no llegan a requerir estudios imaginológicos por esta causa. De hecho, la mayoría de las ASIT en adultos son hallazgos inci- dentales en estudios por patologías no relacionadas como por ejemplo colecistitis o apendicitis.

Son anomalías muy raras, se ha reportado una prevalencia aproximada del Situs Inversus de un $0.01 \%$ de la población de EEUU y en el caso del Situs Ambiguous en el adulto (SAMB) sólo se han publicado pocas series de casos.

A menudo las ASIT y especialmente el SAMB parecen confusas, ya que en la literatura se han usado una variedad de términos distintos para clasificarlas o subclasificarlas tales como síndrome de heterotaxia o simplemente heterotaxia, síndrome de asplenia y de poliesplenia o los diferentes isomerismos ${ }^{(1,2)}$.

Como se desarrollará más adelante, este texto introducirá el uso de la nomenclatura usada en las 
últimas y más importantes publicaciones relacionadas con el tema.

El objetivo de la presente publicación es exponer una revisión bibliográfica, con énfasis en la clasificación de las ASIT del adulto y los hallazgos abdominales en los casos de SAMB. Será complementada con cuatro casos.

Un resumen de los antecedentes, hallazgos y diagnóstico de los pacientes expuestos se puede revisar en la Tabla I.

\section{Terminología}

Como se comentó anteriormente, existe una variedad de términos en la literatura para referirse a las Anomalías del Situs (ASIT) lo cual sumado a su baja frecuencia, ha favorecido la confusión. El presente trabajo se desarrollará en base la terminología y clasificación utilizada por Fulcher y Turner el año 2002 en su publicación en Radiographics ${ }^{(2)}$. Este trabajo presenta una serie de casos de un centro único que incluye SAMB, siendo la de mayor cuantía publicado hasta la fecha con nueve casos en casi treinta años. De igual manera, se propone fomentar el uso en nuestro medio de esta nomenclatura y clasificación para no continuar propagando la ya extensa cantidad de sinónimos para las mismas entidades (Figura 1).

El término "Situs" hace referencia a la posición del corazón, vísceras y grandes vasos en relación a la línea media. De esta manera, Situs Solitus se refiere a la posición normal de los órganos antes descritos, por lo que el ápex cardíaco se sitúa a la izquierda (levocardia) al igual que el bazo y estómago. Por otro lado el hígado, vesícula biliar y vena cava se encuentran a la derecha. También se incluyen en el concepto la correcta posición del colon y las asas de intestino delgado. En estas condiciones la presencia de cardiopatía congénita (CC) es del 1\%(2).

Situs Inversus indica que existe una configuración en espejo en relación a Situs Solitus. Existen dos grandes categorías de Situs Inversus: Situs Inversus con dextrocardia o con levocardia, en donde la variante con dextrocardia, también llamada Situs Inversus Totalis, es ampliamente más frecuente y se presenta con CC en sólo un 3-5\% de los casos, en cambio la variante con levocardia prácticamente todos los casos se presentan con $\mathrm{CC}^{(2)}$.

Situs Ambiguous (SAMB), también llamado heterotaxia o Sd. de heterotaxia, es definido como la posición anormal, ambigua o en la línea media del o de los órganos y vasos, con una disposición distinta a la que se presenta en el Situs Solitus. Se presentan con CC en un $50-100 \%$ de los casos $^{(2)}$. Probablemente ésta es la categoría que se presenta con más problemas al momento de identificar las anomalías y por lo tanto al momento de realizar un correcto diagnóstico y clasificación, debido a que los casos de SAMB no se presentan siempre con el mismo grupo de anormalidades, sino que más bien engloba un espectro de variantes las cuales pueden o no estar presentes, además existiendo distintos grados de afectación. Por otro lado, ninguna de ellas constituye un hallazgo patognomónico.

Existen dos grandes grupos dentro de los SAMB: Con poliesplenia, a veces llamado también Sd. de poliesplenia e isomerismo izquierdo (por presentar sólo dos lóbulos pulmonares en forma bilateral) y con asplenia, Ilamado también Sd de asplenia o isomerismo derecho (por presentar tres lóbulos pulmonares bilateralmente). Cabe destacar que la categoría con asplenia se presenta con CC en un 99-100\% de los casos y además, suelen ser más graves que en las otras $\operatorname{ASIT}^{(1,2)}$.

La clasificación utilizada se resume en la Figura 1.

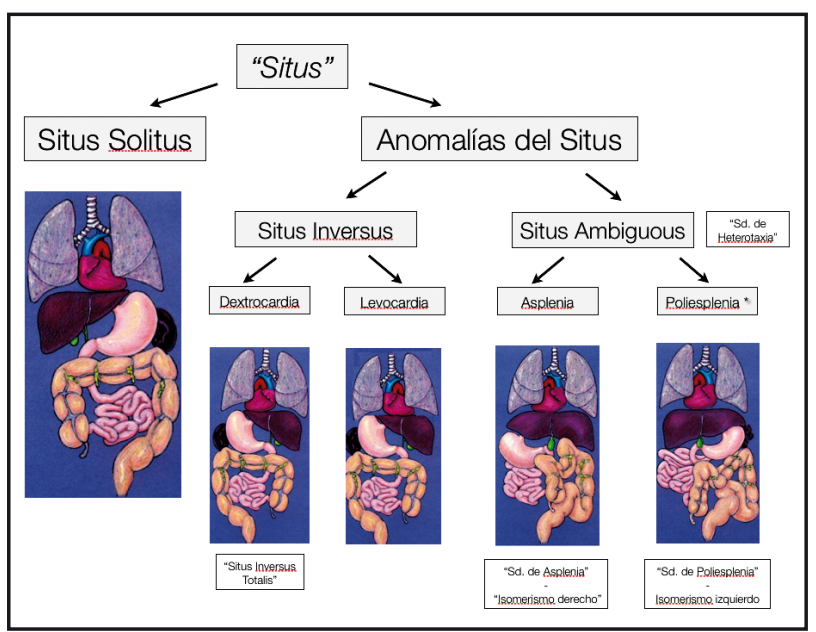

Figura 1. Clasificación de Situs Solitus - Anomalías del Situs y nombres alternativos.

Nota: Los términos en los cuadros trasparentes y entre comillas son sinónimos de la variante correspondiente. Se recomienda evitarlos para evitar confusiones. (Imágenes obtenidas y modificadas de Fulcher $\mathrm{A}$, et $\mathrm{al}^{(2)}$ ).

\section{Hallazgos abdominales de las anomalías del Situs Ambiguous}

Los SAMB pueden presentar un espectro de anormalidades en relación tanto a la ubicación "ambigua" de los órganos, como en las características morfológicas de los mismos. Hay que recordar que no es necesario que todos los órganos estén comprometidos.

Respecto a la posición ambigua de los órganos abdominales, en general, éstos se pueden presentar en una posición normal, en la línea media y continuando el espectro hasta una posición en espejo. Por otro lado, las alteraciones morfológicas son características de cada órgano.

A continuación se describirán los principales hallazgos descritos en la literatura y correlación con los casos expuestos en la Tabla I. 
Tabla I. Casos del Situs Ambiguous.

\begin{tabular}{|c|c|c|c|c|}
\hline Paciente & CASO 1 & CASO 2 & CASO 3 & CASO 4 \\
\hline $\begin{array}{l}\text { Motivo de } \\
\text { consulta }\end{array}$ & $\begin{array}{l}\text { Dolor Abdominal, } \\
\text { Obs. Diverticulitis }\end{array}$ & Asintomático & $\begin{array}{l}\text { Dolor abdominal, } \\
\text { lipasa }(+)\end{array}$ & $\begin{array}{l}\text { Dolor torácico } \\
\text { agudo, Obs. SAA }\end{array}$ \\
\hline Antecedentes & Hombre, 65 años & $\begin{array}{l}\text { Mujer, } 23 \text { años, } \\
\text { asma }\end{array}$ & Mujer, 31 años & $\begin{array}{l}\text { Mujer, } 67 \text { años, } \\
\text { colecistectomía }\end{array}$ \\
\hline $\begin{array}{l}\text { Exámenes } \\
\text { Realizados }\end{array}$ & TC de abdomen & RM de abdomen & $\begin{array}{l}\text { Colangio-RM, TC } \\
\text { de abdomen y pelvis }\end{array}$ & $\begin{array}{l}\text { Angio-TC tórax, } \\
\text { TC de abdomen }\end{array}$ \\
\hline \multirow[t]{7}{*}{$\begin{array}{l}\text { Principales } \\
\text { Hallazgos }\end{array}$} & & $\begin{array}{l}\text { Poliesplenia y } \\
\text { posición en espejo }\end{array}$ & Poliesplenia & Poliesplenia \\
\hline & I VCI-C VA & & I VCI-C VA & I VCl-C VA \\
\hline & Páncreas corto & VCl izquierda & Páncreas corto & Páncreas corto \\
\hline & $\begin{array}{l}\text { Malrotación } \\
\text { intestinal } \\
\text { VB polilobulada }\end{array}$ & $\begin{array}{l}\text { I VCl-C VA } \\
\text { Páncreas corto }\end{array}$ & $\begin{array}{l}\text { Vaso que atraviesa } \\
\text { páncreas }\end{array}$ & $\mathrm{Ml}$ \\
\hline & & & MI & \\
\hline & & $\begin{array}{l}\text { Hígado en línea } \\
\text { media }\end{array}$ & VB polilobulada & \\
\hline & & VB polilobulada & $\begin{array}{l}\text { Vena Porta } \\
\text { preduodenal }\end{array}$ & \\
\hline Diagnóstico & $\begin{array}{l}\text { SAMB con } \\
\text { poliesplenia }\end{array}$ & $\begin{array}{l}\text { SAMB con } \\
\text { poliesplenia }\end{array}$ & $\begin{array}{l}\text { SAMB con } \\
\text { poliesplenia, } \\
\text { Pancreatitis } \\
\text { Aguda Balthazar A }\end{array}$ & $\begin{array}{l}\text { SAMB con } \\
\text { poliesplenia }\end{array}$ \\
\hline \multicolumn{5}{|c|}{$\begin{array}{l}\text { Obs: Observación. } \\
\text { SAA: Sindrome Aórtico Agudo. } \\
\text { TC: Tomografía Computada. } \\
\text { RM: Resonancia Magnética. } \\
\text { IVCI- C VA: Interrupción de la Vena Cava Inferior con Continuación con la Vena Ázigos. VCl: Vena Cava Inferior. } \\
\text { MI: Malrotación Intestinal. } \\
\text { VB: Vesícula Biliar. } \\
\text { SAMB: Situs Ambiguous. }\end{array}$} \\
\hline
\end{tabular}

Bazo: Cuando está presente, puede estar en posición normal, ambigua o en espejo. Presenta especial importancia, ya que en base a los hallazgos morfológicos se subclasifican los SAMB: con poliesplenia y con asplenia (Figura 1). Hacer esta diferencia tiene importancia, ya que los casos de SAMB con asplenia son significativamente menos frecuentes en adultos, dada su alta mortalidad antes del primer año de vida por su mayor asociación con CC tanto en frecuencia como en gravedad y por una inmunidad disminuida. Cabe mencionar que poliesplenia se define como la presencia de dos o más bazos, sin embargo, la presentación característica es con pequeños y múltiples bazos de diferentes tamaños y redondeados también llamados "esplénulos". Es importante destacar que en el caso de presentarse un estudio con características típicas de un SAMB, pero con bazo único, se debe clasificar como SAMB con poliesplenia, sin embargo, esta forma de presentación es poco frecuente ${ }^{(1-3)}$.

Los cuatro casos expuestos presentaron poliesplenia (Tabla I). La variación entre ellos fue de un bazo compuesto por pocos esplénulos (Imagen 1), morfología descrita como característica de la poliesplenia con múltiples esplénulos (Imagen 2) y un caso donde se encontró este órgano en una posición en espejo en el hipocondrio derecho (Imagen 3). 


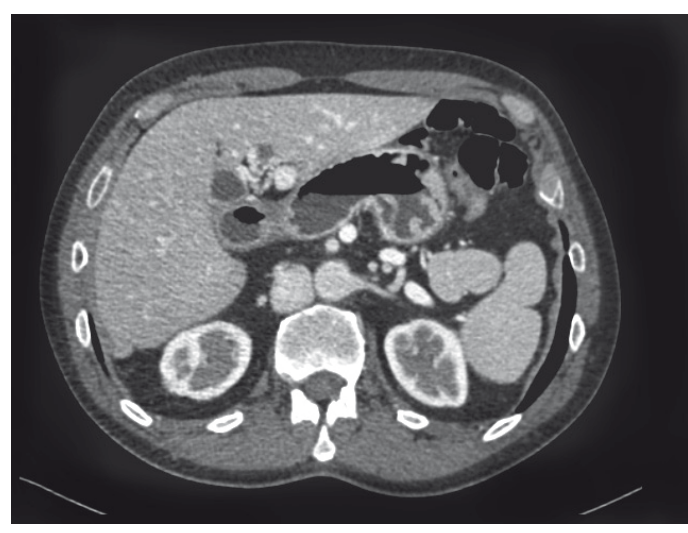

Imagen 1. Corte axial de TC de abdomen en fase portovenosa la cual demuestra una poliesplenia con pocos esplénulos (Caso 1).

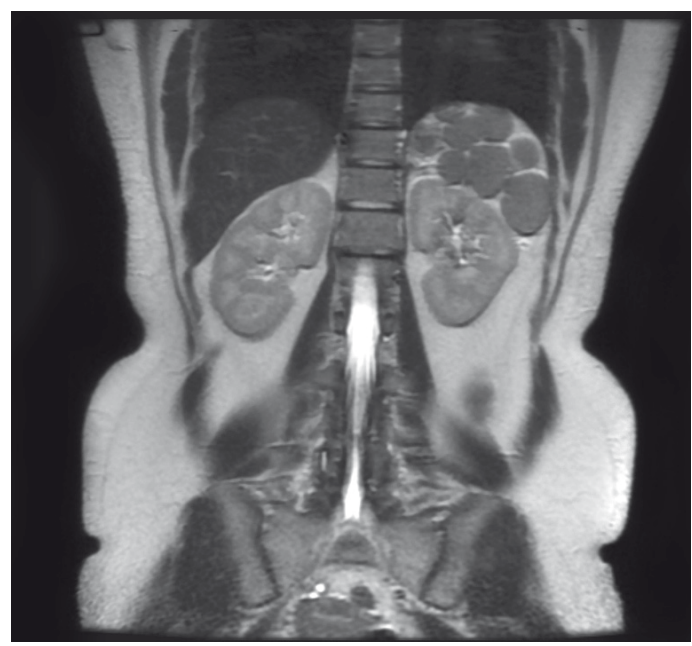

Imagen 2. Corte coronal de RM de abdomen, secuencia potenciada en T2. Demuestra morfología característica de la poliesplenia compuesta por múltiples esplénulos en el hipocondrio izquierdo (Caso 2).

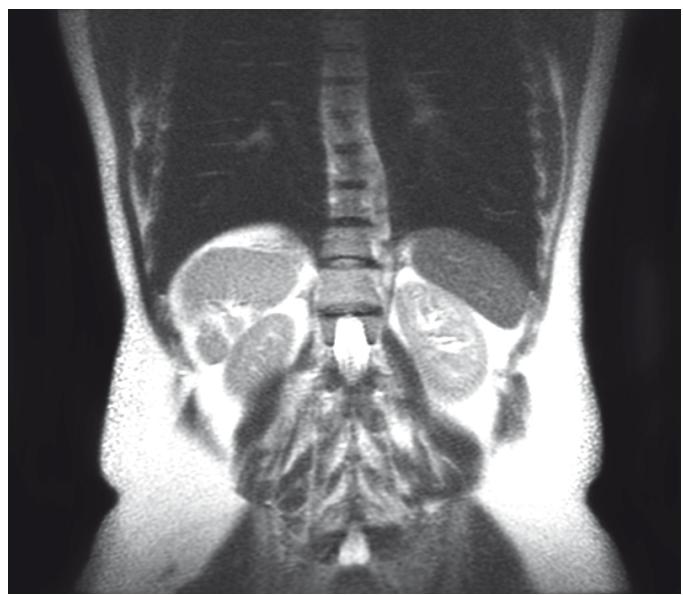

Imagen 3. Corte coronal de RM de abdomen, secuencia potenciada en T2. Se observa poliesplenia de posición en espejo en el hipocondrio derecho (Caso 2).
Hígado, vesícula y vía biliar: los más frecuente son las anomalías de posición, característicamente el hígado y la vesícula biliar se pueden encontrar en la línea media (Imagen 4) o en espejo.

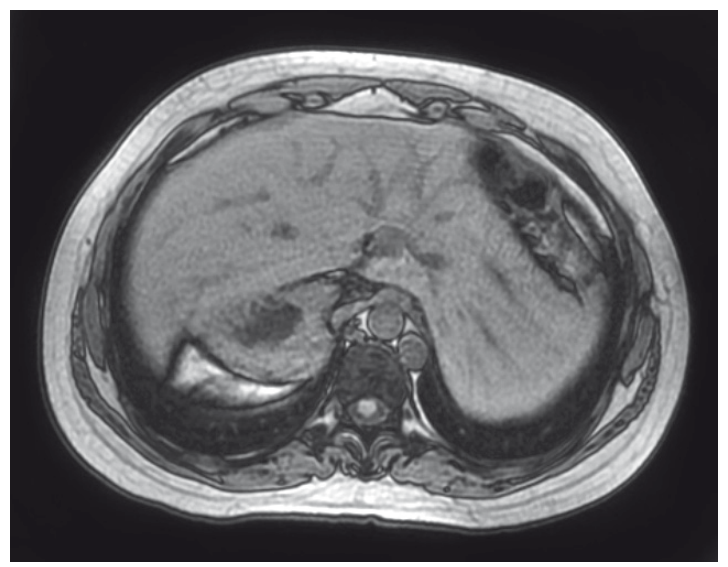

Imagen 4. Corte axial de RM de abdomen, secuencia GRE fuera de fase potenciada en T1. Demuestra la posición del hígado en línea media y del estómago a la derecha (Caso 2).

No se ha descrito un aspecto polilobulado de vesícula biliar en otros estudios, sin embargo, éste fue un hallazgo presente en tres de nuestros casos (Imagen 5).

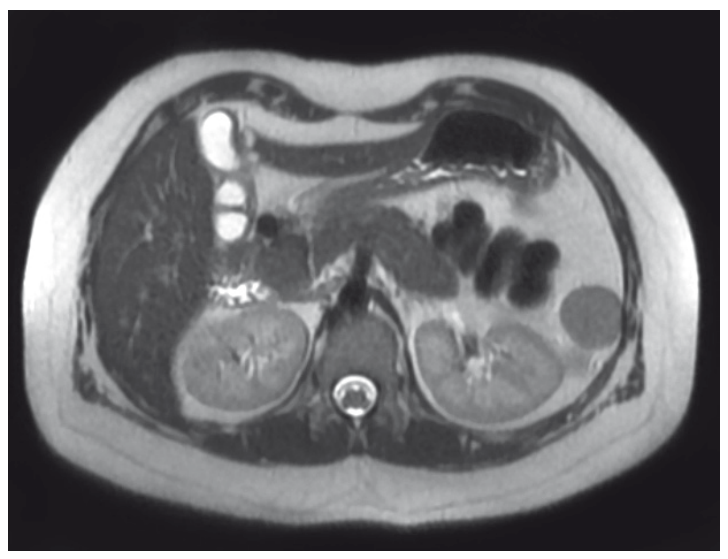

Imagen 5. Corte axial de RM de abdomen, secuencia potenciada en T2. Se observa vesícula biliar de aspecto polilobulado (Caso 3).

Páncreas: como anomalía de posición se describe que la cabeza se encuentra a la derecha de la línea media (Imagen 6). Morfológicamente los SAMB se asocian a páncreas corto (ausencia de cola y/o cuerpo), también a que un vaso puede atravesar la glándula (imagen 7) ${ }^{(2,4)}$. 


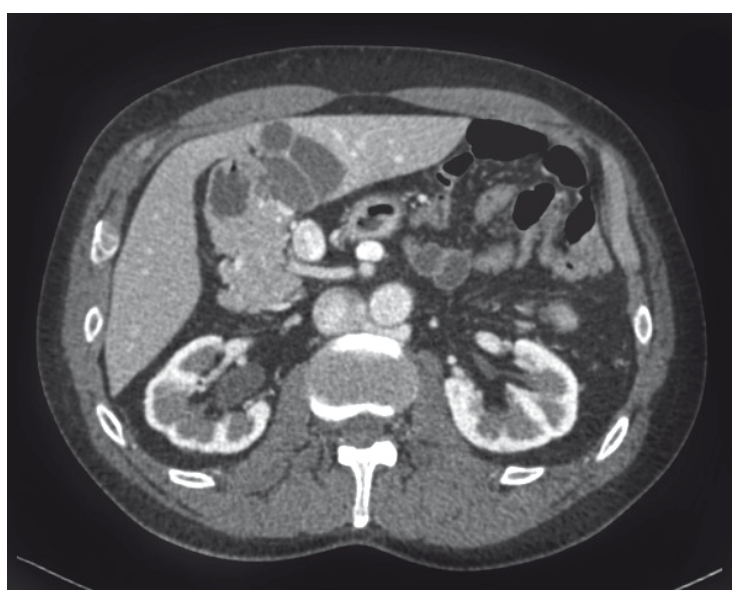

Imagen 6. Corte axial de TC de abdomen en fase portovenosa, demuestra la ubicación de la cabeza del páncreas al lado derecho de la línea media (Caso 1).

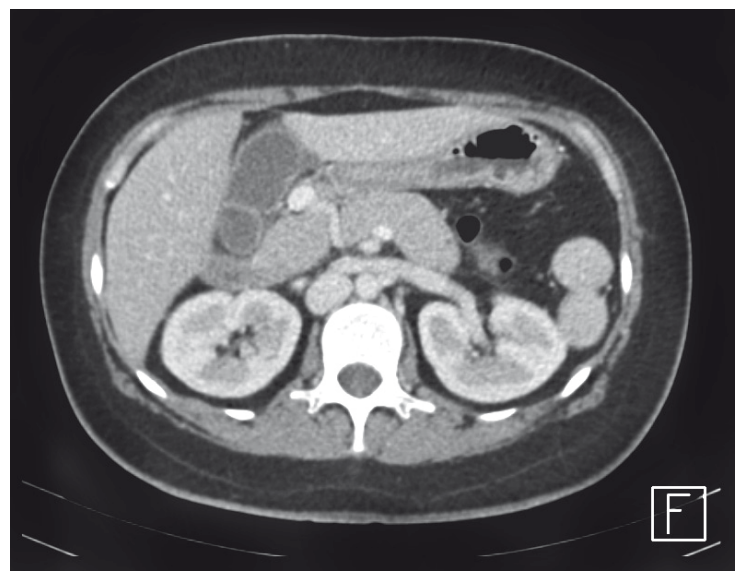

Imagen 7. Corte axial de TC de abdomen en fase portovenosa. Observe el páncreas corto (ausencia de cola) y un vaso que atraviesa la glándula (Caso 3).

Interrupción de la vena cava inferior ( $\mathrm{VCl}$ ) con continuación con vena Ácigos/Hemiácigos: la porción infrahepática de la $\mathrm{VCl}$ se ha descrito en posición normal, a izquierda (Imagen 8) o también que se encuentre duplicada. Frecuentemente no se observa la porción "intrahepática" de la $\mathrm{VCl}$ en el lóbulo caudado y la porción infrahepática se continúa con el sistema ácigos o hemiácigos (Imagen 9); este tipo de anomalía es rara en los pacientes con SAMB con asplenia ${ }^{(1-4)}$.

Todos los casos que reportamos presentaron interrupción de $\mathrm{VCl}$ con continuación con la vena ácigos.

Aorta: se encuentra en su posición normal en la mayoría de los casos descritos. Se ha reportado a la derecha de la línea media cuando coexiste con VCI duplicada, además se han descrito casos donde la $\mathrm{VCl}$ y la aorta se encuentran a un mismo lado ${ }^{(1,2,5)}$.

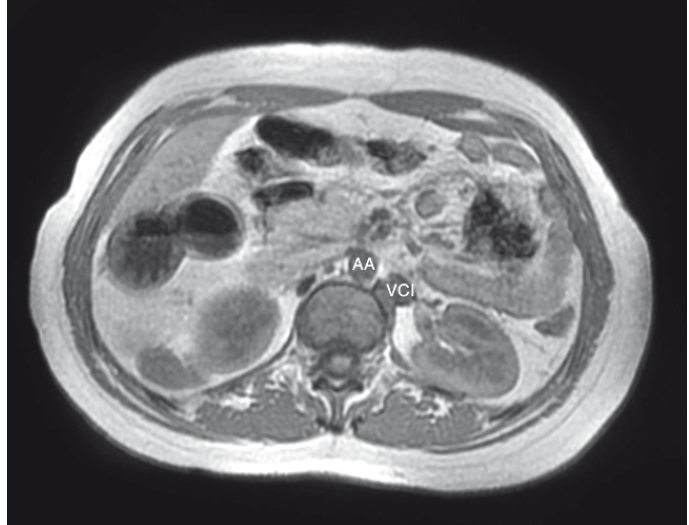

Imagen 8. Corte axial de RM de abdomen, secuencia GRE dentro de fase potenciada en T1. Demuestra la posición de la VCl infrahepática (VCl) a la izquierda de la línea media y de la aorta (AA) (Caso 2).

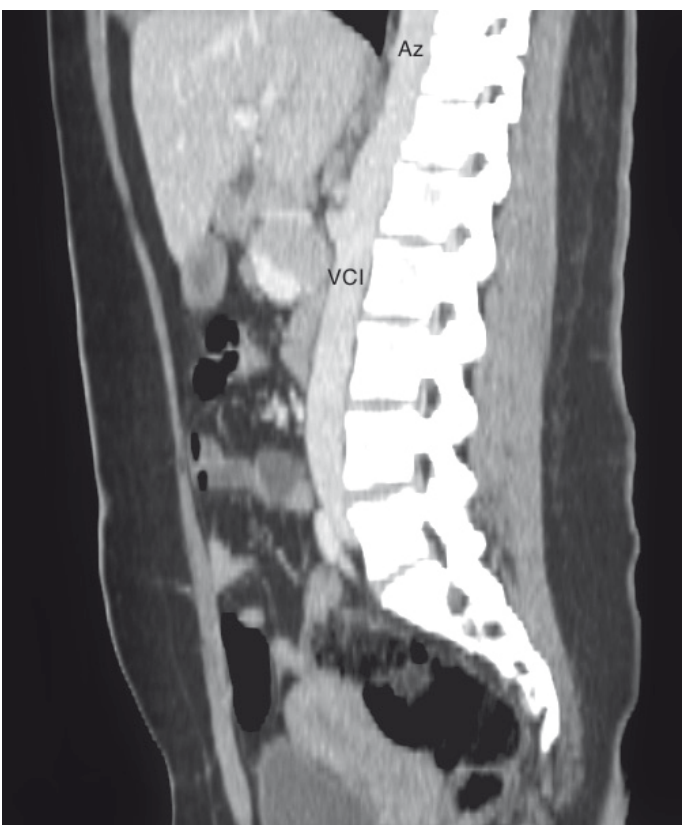

Imagen 9. Corte sagital de TC de abdomen y pelvis en fase porto-venosa, reconstrucción con proyección de intensidad promedio (MIP). Se aprecia la interrupción de la vena cava inferior (VCl) y su continuidad con la vena Ázigos (Az) (Caso 3).

Tubo digestivo: el estómago puede presentar posición normal o en espejo (Imagen 10). Respecto al intestino delgado y colon, se pueden observar distintos grados de malrotación intestinal, siendo lo más descrito que el intestino delgado se encuentre a la derecha de la línea media y el colon a la izquierda (Imagen 11). Otros grados de malrotación se pueden observar, por ejemplo, cuando el ciego no se encuentra completamente fijado al retroperitoneo(2). También se describe en la literatura la posición de la vena porta por delante del duodeno, lo que posiblemente pudiera causar cuadros de obstrucción intestinal parcial (Imagen 12)(4). 


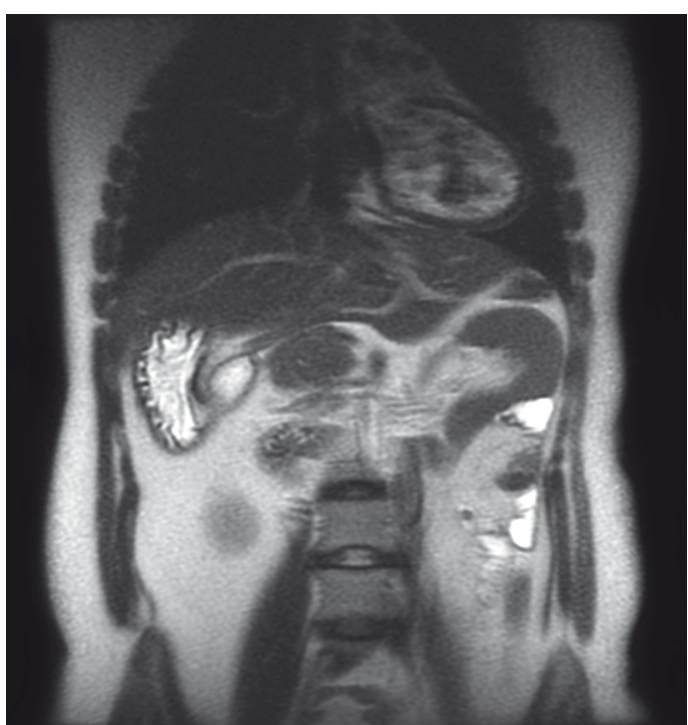

Imagen 10. Corte coronal de RM de abdomen, secuencia potenciada en T2. Se observa el estómago con posición en espejo (Caso 3).

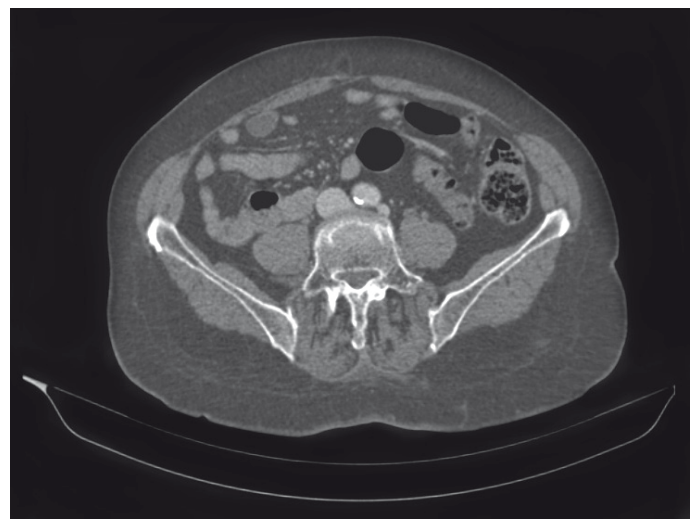

Imagen 11. Corte axial de TC de abdomen en fase portovenosa. Nótese como las asas de colon se encuentran casi exclusivamente a la izquierda de la línea media y como las de intestino delgado a la derecha (Caso 4).

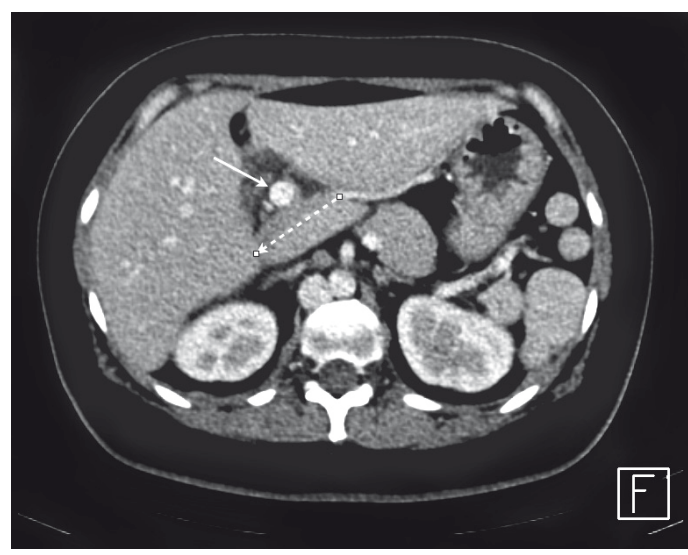

Imagen 12. Corte axial de TC de abdomen en fase portovenosa. Observe como la vena porta (flecha continua) se encuentra en posición pre-duodenal ubicándose anteriormente al duodeno que está colapsado (aspecto superior de flecha discontinua) (Caso 3).

\section{Conclusión}

El diagnóstico y clasificación de los SAMB mediante estudios abdominales puede ser difícil debido a que no se presentan siempre con el mismo grupo ni grado de anormalidades. Por otro lado, dado que históricamente se han utilizado una variedad de términos para referirse a las distintos tipos de ASIT, proponemos consensuar la terminología utilizada en la presente publicación y que se resume en la figura 1, dejando de lado otros términos para evitar confusión.

Actualmente, la mayor disponibilidad de los métodos imaginológicos, nos han demostrado que las ASIT, en especial los tipo SAMB, no son exclusivas de la edad pediátrica y que tampoco están siempre asociadas a comorbilidad, por el contrario, se pueden presentar en adultos y como hallazgo incidental.

Es importante conocer las diferentes variantes e incorporar el concepto de que se trata de un amplio espectro de posibilidades, para poder llegar más fácilmente al diagnóstico y clasificación correcta de la ASIT a la cual nos veamos enfrentados.

\section{Bibliografía}

1. Applegate K, Goske M, Pierce G, Murphy D. Situs Revisited: Imaging of the Heterotaxy Syndrome. RadioGraphics 1999; 19: 837-852.

2. Fulcher A, Turner M. Abdominal Manifestations of Situs Anomalies in Adults. RadioGraphics 2002; 22: 1439-1456.

3. Cronje R, Hugo L, Griessel P. The Association Between Polysplenia, Asplenia and Other Congenital Abnormalities: Organ Isomerism. S Afr Med J 1973; 47: 2264-2266.

4. Plata J, Hernández D, Anthón F, Podgaetz E, Ávila F, Chan C. Polysplenia syndrome in the adult patient. Case report with review of the literature. Ann Hepatol 2004; 3: 114-117.

5. Ruscazio M, Van Praagh S, Marrass A, Catani G, lliceto $S$, Van Praagh R. Interrupted inferior vena cava in asplenia syndrome and a review of the hereditary patterns of visceral situs abnormalities. Am J Cardiol 1998; 81: 111-116. 